\title{
Constructing a biomimetic robust bi-layered hydrophilic lubrication coating on surface of silicone elastomer
}

\author{
Luyao GAO ${ }^{1,2, \uparrow}$, Xiaoduo $\mathrm{ZHAO}^{2, \dagger}$, Shuanhong $\mathrm{MA}^{2, *}$, Zhengfeng $\mathrm{MA}^{2}$, Meirong $\mathrm{CAI}^{2}$, Yong-Min LIANG ${ }^{1, *}$, \\ Feng $\mathbf{Z H O U}^{2}$ \\ ${ }^{1}$ School of Chemistry and Chemical Engineering, Lanzhou University, Lanzhou 730000, China \\ ${ }^{2}$ Lanzhou Institute of Chemical Physics, Chinese Academy of Sciences, Lanzhou 730000, China \\ Received: 28 October 2020 / Revised: 25 December 2020 / Accepted: 30 March 2021 \\ (C) The author(s) 2021.
}

\begin{abstract}
Silicone elastomers-based materials have been extensively involved in the field of biomedical devices, while their use is extremely restricted due to the poor surface lubricity and inherent hydrophobicity. This paper describes a novel strategy for generating a robust layered soft matter lubrication coating on the surface of the polydimethylsiloxane (PDMS) silicone elastomer, by entangling thick polyzwitterionic polyelectrolyte brush of poly (sulfobetaine methacrylate) (PSBMA) into the sub-surface of the initiator-embedded stiff hydrogel coating layer of $\mathrm{P}(\mathrm{AAm}-\mathrm{Co}-\mathrm{AA}-\mathrm{co}-\mathrm{HEMA}-\mathrm{Br}) / \mathrm{Fe}$, to achieve a unified low friction and high load-bearing properties. Meanwhile, the stiff hydrogel layer with controllable thickness is covalently anchored on the surface of PDMS by adding iron powder to provide catalytic sites through surface catalytically initiated radical polymerization (SCIRP) method and provides high load-bearing capacity, while the topmost brush/hydrogel composite layer is highly effective for aqueous lubrication. Their synergy effects are capable of attaining low friction coefficient (COFs) under wide range of loaded condition in water environment with steel ball as sliding pair. Furthermore, the influence of mechanical modulus of the stiff hydrogel layer on the lubrication performance of layered coating is investigated, for which the COF is the lowest only when the modulus of the stiff hydrogel layer well matches the PDMS substrate. Surprisingly, the COF of the modified PDMS could remain low friction $(\mathrm{COF}<0.05)$ stably after encountering 50,000 sliding cycles under $10 \mathrm{~N}$ load. Finally, the surface wear characterizations prove the robustness of the layered lubricating coating. This work provides a new route for engineering lubricious silicon elastomer with low friction, high load-bearing capacity, and considerable durability.
\end{abstract}

Keywords: surface modification; silicone elastomer; layered lubrication coating; hydrogels; polyzwitterionic brush

\section{Introduction}

Organic silicone elastomer-based materials have been widely used in the field of functional biomaterials due to their good mechanical property, biocompatibility, and low manufacturing cost [1-4]. Meanwhile, they are commonly used as biomedical devices such as catheters, drainage tubing, prosthetics, and nose implants [5-9]. However, the inherent surface features of them are always biologically inert and hydro- phobicity, which cannot meet the use requirements as implanted or interventional devices. Especially, the surface of the silicone elastomer-based devices commonly has high friction force when they are used in water-based environment, which inevitably causes pain to the patient. Hence, the main challenge for expanding the application range of silicone elastomer materials is to improve their surface lubrication performance.

In the past 10 years, many strategies have been

\footnotetext{
${ }^{\dagger}$ Luyao GAO and Xiaoduo ZHAO contributed equally to this work.

* Corresponding authors: Shuanhong MA, E-mail: mashuanhong@licp.cas.cn; Yong-Min LIANG, E-mail: liangym@1zu.edu.cn
} 
developed to modify the surface of silicone elastomer materials for reducing the friction coefficient (COF). Among them, surface coating of the hydrophilic polymer layer can effectively improve the water lubrication performance of silicon-based materials surface, especially for catheters and other equipment $[10,11]$. However, the combination between the polymer coating and substrate is commonly noncovalent while the interface bonding force is poor. Furthermore, another way to improve the lubricious property of silicon-based materials is surface grafting polymer brush [12-14]. Although the polymer brush chains and substrate are covalently bonded together, the load-bearing and anti-shearing performances of the traditional brush system is commonly poor at the macro-scale. Fortunately, hydrogels-based materials have attracted much attention in the field of biolubrication due to their high water content along with considerable surface lubricious feature [15, 16]. Therefore, controllable modification of hydrogel layer onto the surface of silicone elastomer is a key mean to improve the lubrication performance of silicone elastomer. For example, Parada et al. and Yu et al. $[17,18]$ suggested a simple strategy to grow lubricious hydrogel layer on the surface of silicone elastomer. Previously, we reported a novel method so-called surface catalytically initiated radical polymerization (SCIRP) to form functional hydrogels lubricious coating on the surface of a variety of materials including silicone elastomer [19]. Although obvious improvement of surface lubricity, the load-bearing capacity of the formed hydrogels layer is very limited due to their poor mechanical strength. Benetti and Spencer, and Dehghani et al. $[20,21]$ reported that the brush hydrogels, which combine the polymer brush with hydrogel, are extremely versatile coatings. Despite the improvement of lubrication performance, the loadbearing property of the brush hydrogels can still be greatly improved if we can extend the thickness of polymer brushes. Recently, our group developed a robust polymer brush-grafted hydrogel composite by chemically embedding hydrophilic polymer brush into the subsurface of the high strength hydrogel [22]. Compared with conventional polymer brush-modified surfaces, the hydrophilic polymer brushes on the surface of the layered material are entangled into the high strength hydrogel network, which allows them to better resist the mechanical sliding-induced shear stresses. Until now, new strategies for generating robust hydrogel lubrication coating on the surface of the inert/hydrophobic elastomer are still urgent.

In this work, we proposed to graft hydrophilic polyelectrolyte brush onto the sub-surface of high strength hydrogel layer for constructing robust layered lubrication coating on the surface of silicone elastomer. The layered lubrication coating is composed by stiff hydrogel layer (load-bearing) and soft brush/hydrogel composite layer (lubrication), for which is covalently bonded onto the surface of silicone elastomer by SCIRP method. Correspondingly, the chemical components, structure, and interface combination strength between polydimethylsiloxane (PDMS) substrate and layered lubrication coating are investigated. The optimal lubrication performance is further explored by regulating the thickness and modulus of the loadbearing hydrogel layer, or can be affected by changing the embedded ATRP initiator concentration and grafting polymerization time of polyzwitterionic polyelectrolyte brush. Finally, the long-time friction test and surface wear characterizations are employed to verify the robustness of layered hydrogel lubrication coating.

\section{Experimental section}

\subsection{Materials}

PDMS base and curing agent (Dow corning), 3(trimethoxysilyl) propyl methacrylate (KH570, Jinan Jinhui Chemical Co.), 2, 2'-bipyridyl (Bipy, > 99.5\%, Shanghai Kefeng Co. Ltd.), acrylamide (AAm, purity 99\%, J\&K Chemical Ltd.), acrylic acid (AA, purity > 99.0\%, Tokyo Chemical Industry Co. Ltd.), N,Nmethylene-bis-acrylamide (MBAA, purity 99\%, SigmaAldrich), potassium persulfate (PPS, purity $>99.6 \%$, Tianjin Chemical Reagents Co.), 2-hydroxyethyl methacrylate (HEMA, purity 99\%, J\&K Chemical Ltd.), 2-bromoisobutyryl bromide (purity 98\%, J\&K Chemical Ltd.), 3-[[2-(methacryloyloxy) ethyl] dimethylammonio] propane-1-sulfonate (SBMA, purity 99\%, Aldrich), and triethylamine (TEA) were purchased and used without any purification. Copper (I) bromide (CuBr) was purified by refluxing in acetic acid. The initiator 
of 2-bromo-2-methylpropionic acid 3-(trichlorosilyl) propyl ester for performing surface-initiated atom transfer radical polymerization (SI-ATRP) was prepared according to the previous work [23].

\subsection{Synthesis of 2-(2-bromoisobutyryloxy) ethyl methacrylate (HEMA-Br)}

The initiator of HEMA-Br was synthesized based on the reported method [24]. $6.25 \mathrm{~g}$ HEMA and $4.05 \mathrm{~g}$ TEA were dissolved into $30 \mathrm{~g}$ dichloromethane in a $100 \mathrm{~mL}$ three-neck round-bottomed flask under $\mathrm{N}_{2}$ protection at $0{ }^{\circ} \mathrm{C}$ for $20 \mathrm{~min}$. $9.164 \mathrm{~g}$ 2-bromoisobutyryl bromide and $15.5 \mathrm{~g}$ dichloromethane were slowly dropped into the above solution. The solution was agitated at $0{ }^{\circ} \mathrm{C}$ for $4 \mathrm{~h}$. The mixture was extracted twice with dichloromethane and filtered to remove white precipitate. The filtrate was treated successively with saturated $\mathrm{Na}_{2} \mathrm{CO}_{3}$ and $\mathrm{NaCl}$ solution for several times until the $\mathrm{pH}$ of solution approaching 7. Finally, the solution was evaporated and refined by column chromatography, using a blend of petroleum ether and acetic ether $(v / v=11: 1)$ as eluent to get a pale-yellow liquid (yield: $60 \%$ ).

\subsection{Preparation of PDMS-KH570-hydrogel samples}

Blending the PDMS base material and its curing agent in a 10:1 weight ratio resulted in a homogeneous and viscous solution. Subsequently, iron powders were added into the above solution to form $50 \%$ black mixture after mechanically stirring for $1 \mathrm{~h}$ at ambient temperature. Followed by bubbles removal, the black mixture was poured into the space between two glass plates and then cured at $80{ }^{\circ} \mathrm{C}$ for $3 \mathrm{~h}$ to obtain Fe-doped PDMS sheets (thickness: $1 \mathrm{~mm}$ ).

The surface of the resulted PDMS sheets were processed by $\mathrm{O}_{2}$ plasma $(98 \mathrm{~W})$ for $60 \mathrm{~s}$. Then the treated sheets were immersed into the activation solution which consisted of $\mathrm{H}_{2} \mathrm{O} / \mathrm{H}_{2} \mathrm{O}_{2} / \mathrm{HCl}(v / v / v=$ 5:1:1) for $3 \mathrm{~min}$. The activated sheets were then soaked in $1.5 \% \mathrm{KH} 570$ ethanol solution for $1 \mathrm{~h}$ to obtain PDMS-KH570 sheets. Next, the PDMS-KH570 sheets were put into the monomer solution to perform SCIRP at room temperature. The monomer solution consisted of AAm, AA, HEMA-Br, MBAA, and PPS. MBAA was used as crosslinking agent and PPS was used as initiator. After polymerization for a certain time, the chemically crosslinked P(AAm-co-AA-co-HEMA-Br) hydrogel layer with controllable thickness was covalently bonded to the surface of PDMS sheets. Then, the P(AAm-co-AA-co-HEMA-Br) hydrogel layercoated PDMS sheets were immersed into $\mathrm{FeCl}_{3}$ solution $(0.25 \mathrm{~mol} / \mathrm{L})$ with different time $(1,12,24$, and $48 \mathrm{~h})$ to form dual-crosslinked P(AAm-co-AA-co-HEMA$\mathrm{Br}) / \mathrm{Fe}$ hydrogel layers. Finally, the P(AAm-co-AAco-HEMA-Br)/Fe hydrogel layer-coated PDMS sheets were immersed into deionized water for $48 \mathrm{~h}$ to remove unreacted components and extra $\mathrm{Fe}^{3+}$, and PDMSKH570-hydrogel samples were successfully obtained.

\subsection{Preparation of PDMS-KH570-hydrogel- PSBMAsamples}

$4 \mathrm{~g}$ SBMA were dissolved into $8 \mathrm{~mL}$ solution of $\mathrm{H}_{2} \mathrm{O} / \mathrm{CH}_{3} \mathrm{OH}(v / v=2: 1)$ and degassed with $\mathrm{N}_{2}$ for 30 min under stirring in a Schlenk tube. Subsequently, $120 \mathrm{mg}$ bipy and $40 \mathrm{mg} \mathrm{CuBr}$ were added to form red-brown reaction solution. Then, the as-prepared PDMS-KH570-hydrogel samples were immersed into the reaction solution to perform ATRP under $\mathrm{N}_{2}$ atmosphere. After reacting for a certain time and then rinsing with deionized water, PSPMA polymer brush was chemically embedded into the sub-surface of the $\mathrm{P}(\mathrm{AAm}-\mathrm{Co}-\mathrm{AA}-\mathrm{co}-\mathrm{HEMA}-\mathrm{Br}) / \mathrm{Fe}$ hydrogel layer, PDMS-KH570-hydrogel-PSBMA samples were obtained.

\subsection{Preparation of control PDMS-PSBMA sample}

Iron-doped PDMS sheets were exposed to $\mathrm{O}_{2}$ plasma $(98 \mathrm{~W})$ for treatment of $1 \mathrm{~min}$. Then the treated PDMS sheets were put into a vacuum desiccator containing of $5 \mu \mathrm{L}$ initiator of (2-bromo-2-methylpropionic acid 3-(trichlorosilyl) propyl ester) to perform chemical vapor deposition of ATRP initiator. In typical case, the desiccator was lowered to a pressure of $<1 \mathrm{mbar}$ and kept under vacuum for 30 min after turning off the pump. After recycling for 3 times, ATRP initiatormodified PDMS sheets were obtained. Next, the initiator-modified PDMS sheets were put into a Schlenk tube containing ATRP reaction solution (the same recipe and components as 2.4) for performing SI-ATRP polymerization under $\mathrm{N}_{2}$ atmosphere. After reacting for $3 \mathrm{~h}$ and rinsing with deionized water, the control PDMS-PSBMA sample was obtained. 


\subsection{Component, morphology, and wettability characterizations}

The surface chemistry composition of PDMS, PDMSKH570, PDMS-KH570-hydrogel, and PDMS-KH570hydrogel-PSBMA were characterized by attenuated total reflection fourier transform infrared spectroscopy (ATR-FTIR, Nicolet iS10, Thermo Scientific, USA) and X-ray photoelectron spectroscopy (XPS, ESCALAB 250Xi, USA). The morphologies of PDMS-KH570hydrogel and PDMS-KH570-hydrogel-PSBMA samples were observed by a scanning electron microscopy (SEM, JSM-5601LV, JEOL, Japan) and the corresponding chemistry elements distribution was analyzed by energy dispersive X-ray spectroscopy (EDS). Fluorescent photographs of PDMS-KH570-hydrogel-PSBMA were characterized by optical microscopy (Olympus, BX51, Japan). The samples for fluorescent imaging were treated with $0.01 \mathrm{~mol} \cdot \mathrm{L}^{-1}$ rhodamine $6 \mathrm{G}$ in advance. The water contact angles (CA) of prepared samples were determined using a DSA-100 optical contact angle meter (Kruss Company, Ltd., Germany). Meanwhile, a droplet of $5 \mu \mathrm{L}$ water was dropped on the surface of test samples at $25{ }^{\circ} \mathrm{C}$ for every test. The average $\mathrm{CA}$ value was obtained by measuring at least three different positions on the sample.

\subsection{Mechanical property measurement}

The interfacial adhesion between hydrogel layer and PDMS sheet was accessed by employing $90^{\circ}$ peeling test at an electrical universal material testing machine (EZ-Test, SHIMADZU, Japan) with a $500 \mathrm{~N}$ load cell. The tests were carried out at a rate of $30 \mathrm{~mm} \cdot \mathrm{min}^{-1}$ and the width of samples was fixed at $10 \mathrm{~mm}$. The test data was repeated as a minimum of five times and averaged.

The elastic modulus of the hydrogel layer was measured by separating the hydrogel from PDMS substrate after performing SCIRP without KH570 treatment. In detail, the $\mathrm{P}(\mathrm{AAm}-\mathrm{co}-\mathrm{AA})$ hydrogel was generated on the surface of the PDMS substrate. After growing for $20 \mathrm{~min}$, the hydrogel layer was peeled off from the substrate and was immersed into the $\mathrm{FeCl}_{3}$ solution for different time $(1,12,24$, and $48 \mathrm{~h})$ to obtain hydrogel sheet with different elastic modulus. The tensile test was employed to obtain the elastic modulus of the hydrogel layer. The tensile tests were performed at a velocity of $50 \mathrm{~mm} / \mathrm{min}$. The elastic modulus was determined from the slope $2 \%-5 \%$ of the strain ratio of the stress-strain curve. Each experiment was at least repeated five times.

\subsection{Friction test and wear analysis}

The COFs were measured by using a conventional reciprocating tribometer at a ball-on-disk mode (CSM Co. Ltd., Switzerland). The friction pairs were 316L stainless balls (diameter: $6 \mathrm{~mm}$ ), the frequencies are $0.05,0.5,1.0,2.0$, and $5.0 \mathrm{~Hz}$, the amplitude is $5 \mathrm{~mm}$, the loads are $1,2,5,10$, and $20 \mathrm{~N}$, the lubricant is water. After friction test, the wear scars of the materials were characterized using a surface three-dimensional (3D) mapping profiler (MicroXAM-800, USA) with the non-contact diffraction mode immediately to obtain the wear area. The cross-sectional area of the wear scar was calculated by the software of the surface 3D mapping profiler. Subsequently, the samples were frozen in liquid nitrogen and then freeze-dried at -30 ${ }^{\circ} \mathrm{C}$ in $1 \mathrm{~Pa}$ for $24 \mathrm{~h}$. Finally, the samples were sprayed gold three times and observed by SEM to obtain the surface wear morphologies.

\subsection{Statistical analysis}

All values were reported with the standard deviation of the mean. All data were analyzed using one-way ANOVA and then tested for normality. The calculated results were made by the Tukey test with statistical significance evaluated at $p<0.05$.

\section{Results and discussion}

\subsection{Preparation and characterizations}

The preparation process of PDMS-KH570-hydrogelPSBMA samples is schematically illustrated in Fig. 1. Firstly, the surface of PDMS sheets doped with iron powder catalysts was treated by a series of surface modification strategies for chemically grafting KH570 onto it. The purpose of adding iron powder is to provide catalytic site on PDMS surface. Then, one layer of chemically crosslinked P(AAm-co-AA-co-HEMA$\mathrm{Br}$ ) hydrogel layer with controllable thickness was 


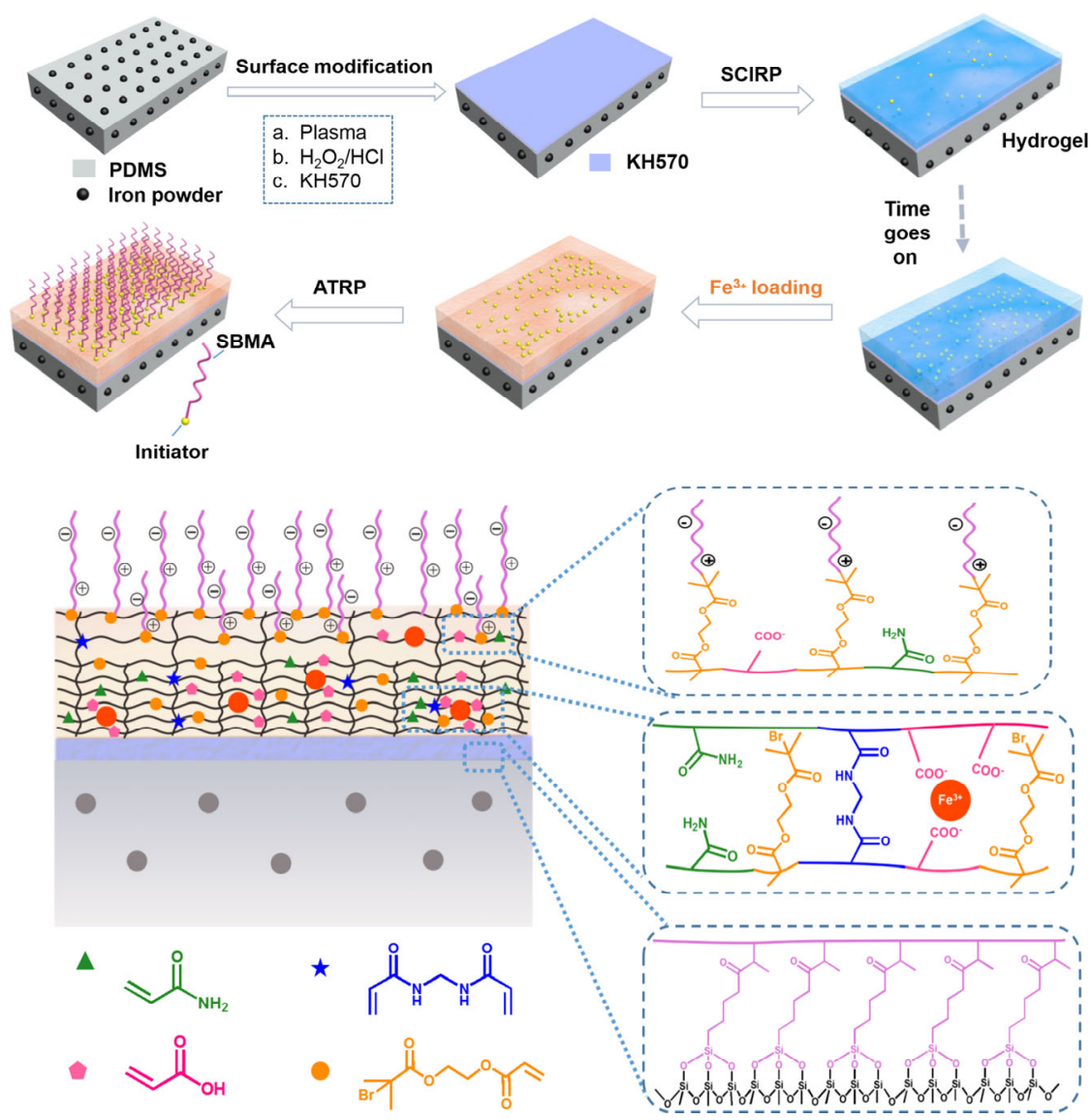

Fig. 1 The schematic illustration for the preparation procedures and the structure of PDMS-KH570-hydrogel-PSBMA.

generated on the surface of the treated PDMS sheets by SCIRP method [19], based on the well-known redox mechanism between $\mathrm{Fe}^{2+}$ and $\mathrm{S}_{2} \mathrm{O}_{8}{ }^{2-}$. Meanwhile, the ATRP initiator of HEMA-Br was chemically embedded within the entire hydrogel network, while KH570 acted as a bridge to enhance the interfacial adhesion between the PDMS sheets and hydrogel layer. Next, the PDMS-KH570-hydrogel samples were soaked into $\mathrm{FeCl}_{3}$ solution to form dual-crosslinked hydrogel network layer of $\mathrm{P}(\mathrm{AAm}-\mathrm{co}-\mathrm{AA}-\mathrm{co}-\mathrm{HEMA}-\mathrm{Br}) / \mathrm{Fe}$. Finally, the polymer brush/hydrogel composite lubrication layer were generated on the sub-surface of the $\mathrm{P}(\mathrm{AAm}-\mathrm{Co}-\mathrm{AA}-\mathrm{co}-\mathrm{HEMA}-\mathrm{Br}) / \mathrm{Fe}$ hydrogel layer by performing the ATRP.

In order to characterize the surface components of the samples, ATR-FTIR was utilized to analyze the surface characteristic absorption peaks of the asprepared samples. As shown in Fig. 2(a), compared with bare PDMS-KH570, the appeared absorption peaks of PDMS-KH570-hydrogel sample at 3,185 $\mathrm{cm}^{-1}$ and $1,601 \mathrm{~cm}^{-1}$ can be assigned to $\mathrm{N}-\mathrm{H}$ stretching vibration and blending vibration in AAm of $\mathrm{P}(\mathrm{AAm}-$ co-AA-co-HEMA-Br) hydrogel layer. For PDMSKH570-hydrogel-PSBMA sample, the obvious $-\mathrm{S}=\mathrm{O}$ stretch vibration peak at $1,167 \mathrm{~cm}^{-1}$ was observed, implying the successful grafting of PSBMA polymer brush. Furthermore, XPS was employed to characterize the surface chemical components. Compared with bare PDMS, the appearance of $\mathrm{Br} 3 \mathrm{~d}$ at $70 \mathrm{eV}$ of PDMSKH570-hydrogel sample indicated the ATRP initiator was successfully embedded within the hydrogel network (Fig. 2(b)). As shown in Fig. 2(c), the appeared $\mathrm{N}$ 1s peak at $399.7 \mathrm{eV}$ of PDMS-KH570-hydrogel sample might be ascribed to PAAm in P(AAm-coAA-co-HEMA-Br) hydrogel layer. After grafting the PSBMA polymer brush, the $\mathrm{N}$ 1s peak changed from a single peak to double peaks. The new peak appeared at $402.6 \mathrm{eV}$ was attributed to $\mathrm{NR}_{4}{ }^{+}$in PSBMA. Besides, the $S 2 p$ peak appeared at $167.5 \mathrm{eV}$ and $\mathrm{Br} 3 \mathrm{~d}$ peak disappeared at $70 \mathrm{eV}$ also prove that polymer brush was successfully grafted onto the surface of PDMS-KH570-hydrogel sample. Subsequently, the 

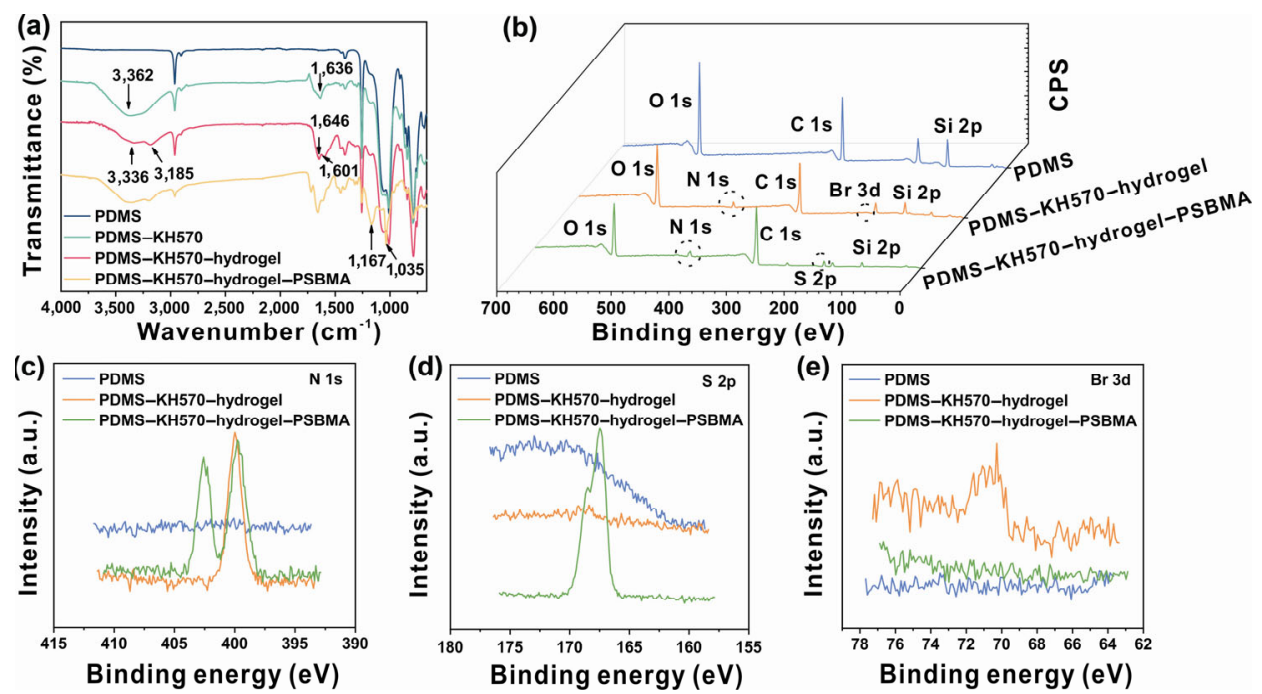

Fig. 2 (a) ATR-FTIR spectra of PDMS, PDMS-KH570, PDMS-KH570-hydrogel, and PDMS-KH570-hydrogel-PSBMA. (b) XPS analysis of the chemical composition of PDMS, PDMS-KH570-hydrogel, and PDMS-KH570-hydrogel-PSBMA. Characteristic element of (c) N 1s, (d) S 2p, and (e) Br 3d for PDMS, PDMS-KH570-hydrogel, and PDMS-KH570-hydrogel-PSBMA, respectively.

surface wettability of the bare PDMS sheet, PDMSKH570-hydrogel sample and PDMS-KH570-hydrogelPSBMA sample was evaluated. Figure S1 in the Electronic Supplementary Material (ESM) shows the water CAs for all samples at different stages of the modification process. Since PSBMA polymer brush is super hydrophilic, the surface CA of PDMS-KH570hydrogel sample after grafting PSBMA polymer brush was significantly reduced.

As shown in Fig. 3(a), typical three-layered structure of the PDMS-KH570-hydrogel-PSBMA sample (the growth time of the hydrogel was $5 \mathrm{~min}$ and the polymer brush grafting time was $120 \mathrm{~min}$ ) was clearly identified by the cross-sectional SEM image. The bottom layer was PDMS substrate doped with a certain amount of iron powder, the middle layer was the remained dual-crosslinking $\mathrm{P}(\mathrm{AAm}-\mathrm{CO}-\mathrm{AA}-\mathrm{co}-\mathrm{HEMA}-\mathrm{Br}) / \mathrm{Fe}$ hydrogel layer after grafting PSBMA polymer brush, and the top level was the composite layer of hydrogel and PSBMA polymer brush. Correspondingly, Fig. S2 in ESM presented the cross-sectional SEM images of PDMS-KH570-hydrogel-PSBMA samples for other hydrogel growth time. With extending the hydrogel growth time, it was found that the thickness of the top composite layer also increased. The growth kinetics for middle hydrogel layer and top composite layer of PDMS-KH570-hydrogel-PSBMA sample was investigated systematically. As shown in Figs. 3(b) and $3(\mathrm{c})$, the thickness of middle hydrogel layer and total thickness both increased with the prolongation of hydrogel growth time. In typical case, when hydrogel growing for 3-20 min, the thickness of hydrogel layer presented a steady rising from 166 to $240 \mu \mathrm{m}$ (Fig. 3(c)), while the thickness was close to $600 \mu \mathrm{m}$ when the growth time of hydrogel was $30 \mathrm{~min}$. Correspondingly, the total thickness of middle hydrogel layer and top composite layer could reach 1,298 $\mu \mathrm{m}$ when the hydrogel growth time was $30 \mathrm{~min}$. As shown in Fig. 3(d), the cross-sectional morphologies of PDMSKH570-hydrogel-PSBMA sample with different PSBMA grafting time under the same hydrogel growth time (20 min) can be intuitively observed by the fluorescent images. As expected, the top polymer brush/ hydrogel composite layer increased in thickness with extending the polymerization time for generating PSBMA brush/hydrogel composite layer. When polymerization time for grafting PSBMA polymer brush was $30 \mathrm{~min}$, the thickness of the top composite layer only was $102 \mu \mathrm{m}$, while its thickness could reach $250 \mu \mathrm{m}$ when polymerized for $60 \mathrm{~min}$. Finally, the thickness of the top composite layer could reach $770 \mu \mathrm{m}$ when the reaction lasted for $240 \mathrm{~min}$. Figure 3(e) shows the element distribution of the three-layered structure of the PDMS-KH570-hydrogel-PSBMA sample. The appearance of obvious signal for characteristic element $\mathrm{S}$ in top polymer brush/hydrogel composite layer further proved the successful grafting of PSBMA brush. 

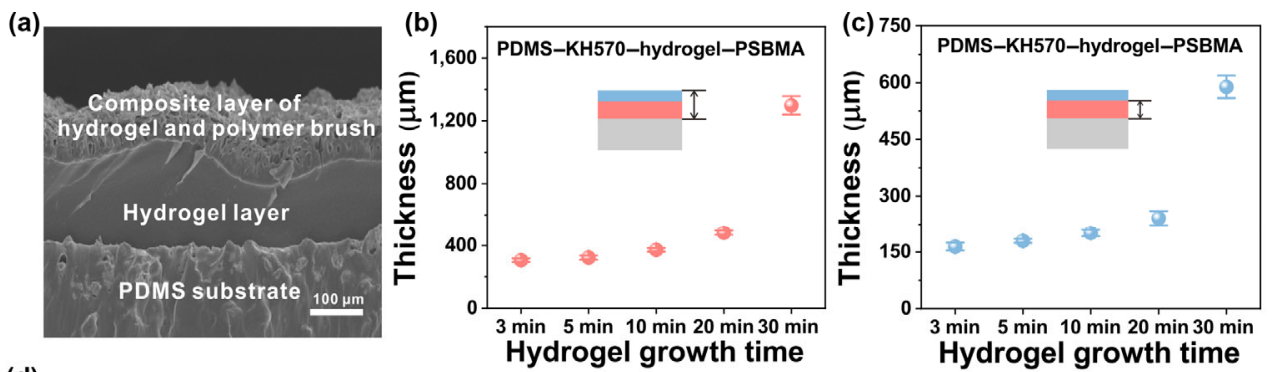

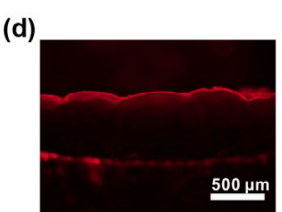

$5 \mathrm{~min}$

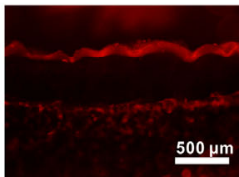

$30 \mathrm{~min}$

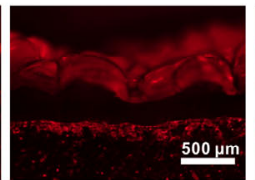

$60 \mathrm{~min}$

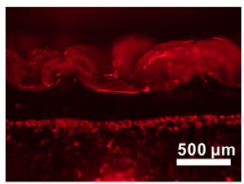

$120 \mathrm{~min}$

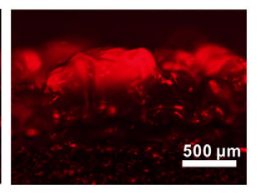

$240 \mathrm{~min}$
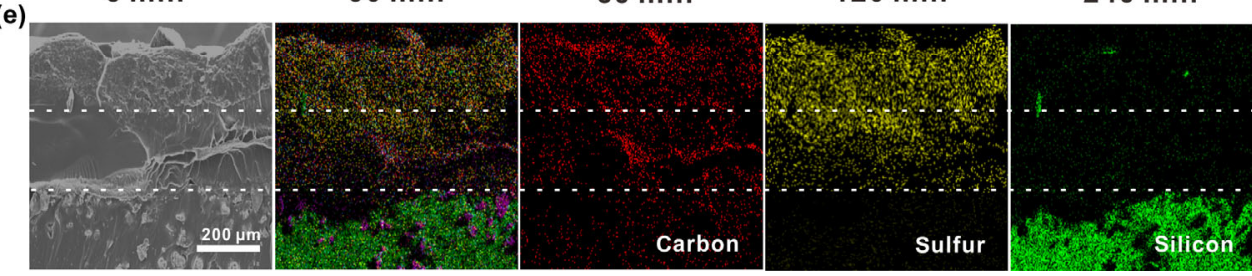

Fig. 3 (a) Cross-sectional SEM image of PDMS-KH570-hydrogel-PSBMA sample (growth time for generating P(AAm-co-AAco-HEMA-Br) hydrogel layer was $5 \mathrm{~min}$ and the polymerization time for generating PSBMA brush/hydrogel composite layer was 120 min); (b) total thickness of the remained middle hydrogel layer and the top polymer brush/hydrogel composite layer versus hydrogel growth time (polymerization time for generating PSBMA brush/hydrogel composite layer was 120 min); (c) thickness of remained middle hydrogel layer versus hydrogel growth time (polymerization time for generating PSBMA polymer brush was 120 min); (d) cross-sectional fluorescence images of PDMS-KH570-hydrogel-PSBMA sample with different polymerization time for grafting PSBMA polymer brush (growth time for generating P(AAm-co-AA-co-HEMA-Br) hydrogel layer was fixed at 20 min); and (e) cross-sectional SEM image of PDMS-KH570-hydrogel-PSBMA sample and corresponding EDS mapping (growth time of hydrogel was $20 \mathrm{~min}$ and polymerization time for generating PSBMA brush/hydrogel composite layer was $120 \mathrm{~min}$ ).

\subsection{Evaluation of interface combination force between hydrogel layer and PDMS sheet}

$90^{\circ}$ peeling test was performed to quantify the interfacial adhesion between the hydrogel layer and the PDMS sheet, as shown in Fig. 4(a). The results showed that
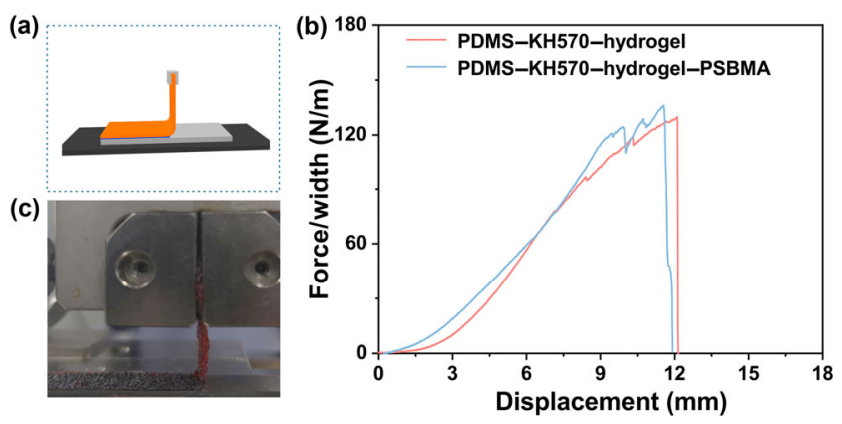

Fig. 4 (a) Schematic depiction of the peel test process; (b) force/ width versus displacement curve of the mechanical peel test for PDMS-KH570-hydrogel and PDMS-KH570-hydrogel-PSBMA; and (c) photograph during the peel test of PDMS-KH570hydrogel-PSBMA. the interface binding force didn't depend on whether polymer brush was grafted or not. For PDMS-KH570hydrogel samples, the interface binding force between hydrogel layer and elastomer could be $129.93 \mathrm{~N} \cdot \mathrm{m}^{-1}$. After grafting polymer brush, the interface binding force for PDMS-KH570-hydrogel-PSBMA samples could be $136.12 \mathrm{~N} \cdot \mathrm{m}^{-1}$, which was a little higher than that of samples without polymer brush. As a result, polymer brush has little influence on the interface adhesion for the hydrogel layer and substrate.

\subsection{Evaluation of the lubrication performance}

Firstly, the variation of COFs of PDMS-KH570hydrogel-PSBMA with different hydrogel growth time are presented in Fig. 5(a). The growth time of the hydrogel had a significant impact on the COFs. It was known that with the same polymerization time for generating PSBMA brush/hydrogel composite layer, the total thickness of the composite layer 

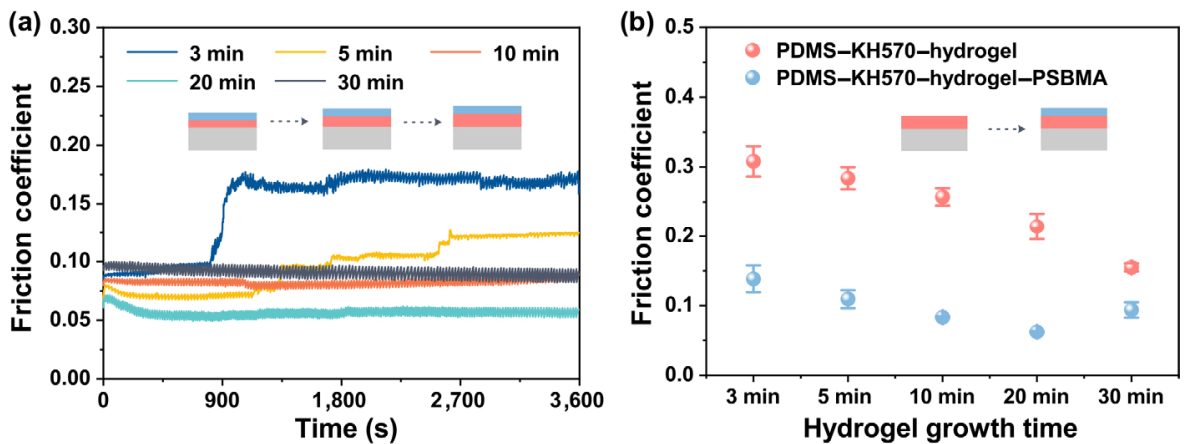

Fig. 5 (a) Variation of dynamic COFs for the PDMS-KH570-hydrogel-PSBMA samples with different hydrogel growth time and (b) the average COFs of PDMS-KH570-hydrogel and PDMS-KH570-hydrogel-PSBMA with different hydrogel growth time (growth time of polymer brush: $120 \mathrm{~min}$; applied load: $10 \mathrm{~N}$; frequency: $1 \mathrm{~Hz}$; and duration: $60 \mathrm{~min}$ ).

(hydrogel and polymer brush) increased with time of hydrogel growth extension (Fig. S2 in ESM), and in terms of friction behavior, with hydrogel growth time prolonging from 3 to $20 \mathrm{~min}$, the COF gradually decreased from 0.139 to 0.063 (Fig. 5(a)). It was worth noting that as long as the growth time of the hydrogel was $30 \mathrm{~min}$, its COF increased rather than decreased. That abnormal phenomenon might be due to the increased elastic deformation resistance and rising contact area. With the same time of grafting polymer brush, the thickness of composite layer for hydrogel and polymer brush increased as hydrogel growth time prolonging (as shown in Fig. S2 in ESM). When the thickness of composite layer was too thick, closer to the millimeter scale, it caused a large elastic deformation on the contact surface under heavy load, which made the contact depth of the friction pair in the lubricant layer increase. Consequently, it induced a larger true contact area, resulting in the increase of COFs. So, the COF of PDMS-KH570-hydrogelPSBMA increased abnormally.

Figure 5(b) shows the evolution of mean COFs for PDMS-KH570-hydrogel and PDMS-KH570-hydrogelPSBMA with different hydrogel growth time. In general, with the same hydrogel growth time, the introduction of PSBMA brush onto the PDMS-KH570-hydrogel surface resulted in a significant reduction of the COF. It could be seen that when the growth time of the hydrogel was $3 \mathrm{~min}$, the COFs of the PDMS-KH570hydrogel-PSBMA and the PDMS-KH570-hydrogel were 0.139 and 0.308 , respectively. For other hydrogel growth time, the COFs for the two as-prepared samples had the same trend as that at $3 \mathrm{~min}$. This is in accordance with the results obtained in several studies which attributed the friction reducing action of polyzwitterionic brush to their boundary lubricating characteristics under water lubrication [25-29]. The polyzwitterionic brush could combine a large number of water molecules to form an effective lubricating protective film, which could prevent direct contact between the sliding surfaces, thus effectively reducing the interface friction [30-32]. Overall, the above results suggest that the surface modification treatment of PDMS can successfully reduce the COFs at an optimized hydrogel growth time (20 min).

Seeking an appropriate polymerization time to construct a polymer brush film is a very important factor for long-term application of the hydrogel composite layer, Fig. 6(a) reveals the effect of the polymerization time for PDMS-KH570-hydrogelPSBMA samples on their friction properties. With the increase of polymerization time (5-120 $\mathrm{min})$, the COF showed a trend of decreasing with the increasing of the thickness of the composite layer (Fig. 3(d)). It was well known that polyzwitterionic brush was capable of stabilizing the surrounding water molecules and forming a liquid-like hydration layer, and the thickness of the hydration layer would increase in accordance with polymer brush layer thickening, thereby reducing the $\mathrm{COF}$ at the tribo-pair interface.

However, when the polymerization time was 240 min, the obtained PDMS-KH570-hydrogel-PSBMA sample started to experience an increase of COF. The thickness of the polymer brush layer on the surface was relatively large at that condition, and the change in the COF could be explained by the increased elastic 

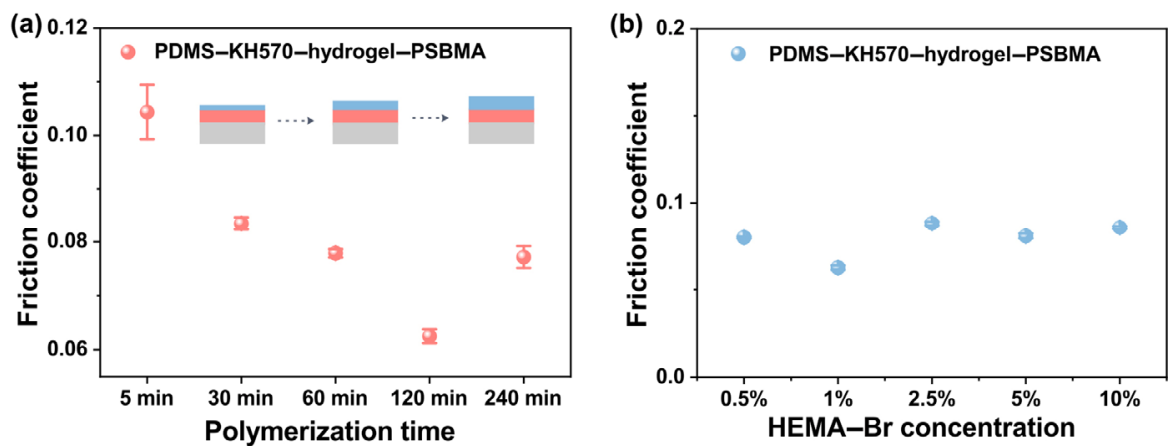

Fig. 6 The average COFs for (a) PDMS-KH570-hydrogel-PSBMA samples with different polymerization time for generating PSBMA brush/hydrogel composite layer and (b) PDMS-KH570-hydrogel-PSBMA samples with different initiator agent HEMA-Br concentration (growth time of the hydrogel: $20 \mathrm{~min}$; load: $10 \mathrm{~N}$; frequency: $1 \mathrm{~Hz}$; duration: $60 \mathrm{~min}$ ).

deformation and rising true contact area. During the friction test, the stainless steel ball was pressed into the polymer brush layer, which increased the contact area between the tribo-pair. In addition, part of the polymer brush could be consumed by shearing. Thus, it could be seen from the above results that the optimum polymerization time for generating polyzwitterionic polyelectrolyte brush was $120 \mathrm{~min}$.

The friction behaviors of the PDMS-KH570hydrogel-PSBMA samples with various concentration of initiator agent (HEMA-Br) are illustrated in Fig. 6(b). Under the premise of the same hydrogel, when there was no initiator HEMA-Br in system, the COF of PDMS-KH570-hydrogel was 0.225. However, it was interesting to note that the COF decreased significantly when there was a small amount of initiator in the system. When the concentration of HEMA-Br was $0.5 \%$, the COF of PDMS-KH570-hydrogel-PSBMA was only 0.080 . But the level of initiator concentration had limited influence on the COF of PDMS-KH570-
hydrogel-PSBMA. Compared with other concentrations, when HEMA-Br was 1\%, the COF of PDMSKH570-hydrogel-PSBMA was relatively lowest at 0.063 . Hence, in consideration of economy, 1\% HEMA$\mathrm{Br}$ was used as the optimal initiator concentration for the following tests.

We further tested the effect of elastic modulus of samples on the friction properties of the materials (Fig. 7). By constructing the coordination interaction between $\mathrm{Fe}^{3+}$ and $\mathrm{COO}^{-}$, after immersing the sample in the ferric chloride solution for different times, materials with different mechanical properties could be obtained [33]. The elastic modulus of hydrogel layer is shown in Fig. 7(a), and the modulus of elasticity of the hydrogel layer improved as the time of immersion in $\mathrm{Fe}^{3+}$ solution increased (Fig. 7(a)). When the soaking time is $24 \mathrm{~h}$, the elastic modulus of the obtained hydrogel $(2.97 \mathrm{MPa})$ is similar to that of PDMS itself (2.84 MPa).

As shown in Fig. 7(b), when the elastic modulus of
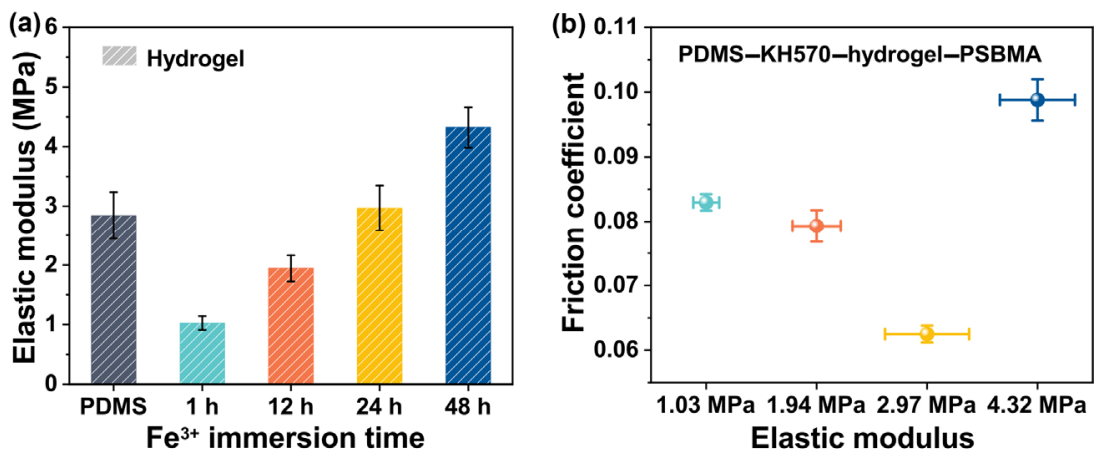

Fig. 7 (a) Representative elastic modulus of the hydrogel layer after immersing in $\mathrm{Fe}^{3+}$ solution for different time and the elastic modulus of PDMS; (b) COFs of PDMS-KH570-hydrogel-PSBMA as a function of elastic modulus for different hydrogel layers (The growth time of the hydrogel: $20 \mathrm{~min}$; the polymer brush grafting time: $120 \mathrm{~min}$; the concentration of initiator in samples: $1 \%$; load: $10 \mathrm{~N}$; frequency: $1 \mathrm{~Hz}$; duration: $60 \mathrm{~min}$ ). 
hydrogel layer of the sample was similar to that of PDMS, they both had similar resistance to elastic deformation, and a lowest COF (0.063) was obtained. For the material that the elastic modulus of hydrogel layer was more than that of the PDMS layer, the PDMS layer could make larger elastic distortion than the hydrogel layer in the process of friction. So, on the view of macroscopic, the COF of material was higher than other circumstances (Fig. 7(b)).

Later, the effects of frequency and load on the COF of the PDMS-KH570-hydrogel-PSBMA were also examined. As illustrated in Fig. 8(a), when the applied load was $10 \mathrm{~N}$, the COFs of the samples showed a trend of first decreasing and then increasing with the increase of sliding frequency, the lowest and the highest COFs were 0.063 and 0.078 , respectively. These results indicated that the changes in frequency had no significant bearing on the COF of PDMS-KH570hydrogel-PSBMA. When the friction frequency was fixed at $1 \mathrm{~Hz}$, their COFs gradually increased from 0.040 to 0.099 as the applied load increased (Figs. 8(c) and $8(\mathrm{~d})$ ). The interface contact stresses under different loads were calculated by classical Hertz contact theory (Fig. S3 in ESM). The increase of the COF was caused by the increase of the embedded depth of the friction pair with the increase of the applied load, resulting in the increase of the elastic deformation of the lubrication layer. Compared with frequency, the applied load had more influence on the friction performance of PDMS-KH570-hydrogel-PSBMA.

\subsection{Comparison with traditional modification system and long-time friction test}

The friction properties of untreated and treated PDMS samples are shown in Fig. 9(a). For PDMS without any modification, the effect of friction reduction was not seen from the experimental results. In addition, the COF of bare PDMS substrate was as high as 0.9 under $5 \mathrm{~N}$ load. After modified with zwitterionic polymer brush, the PDMS-PSBMA displayed no lubrication performance due to polymer brush was easily worn away by steel ball. On the contrary, under the same condition, the COF of PDMS-KH570-hydrogel-PSBMA was only 0.052 . In contrast, the COF of PDMSKH570-hydrogel was relatively high as 0.204 . It had been mostly attributed to the low friction performance of polymer brush in water environment. To sum up, the PDMS-KH570-hydrogel-PSBMA exhibited good lubrication performance.

Next, with optimum growth time of hydrogel (20 min), HEMA-Br concentration (1\%), polymerization time for generating polyzwitterionic polyelectrolyte brush (120 min), and immersion time (24 h), the PDMSKH570-hydrogel-PSBMA was conducted for 50,000 friction cycles at $10 \mathrm{~N}$ load as displayed in Fig. 9(b). During the 50,000 cycles, there was no sudden surge
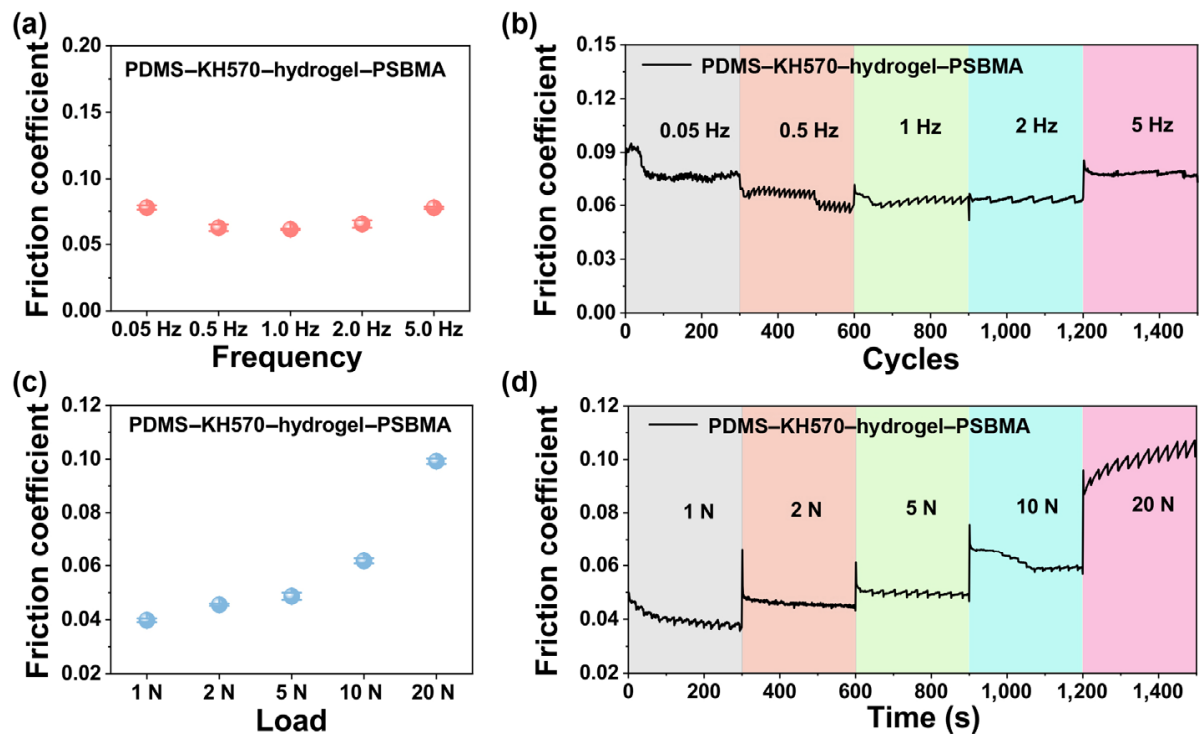

Fig. 8 Influence of test conditions including ( $a, b)$ sliding frequency (applied load: $10 \mathrm{~N}$ ) and (c, d) normal load (reciprocating frequency: $1 \mathrm{~Hz}$ ). The growth time of hydrogel and grafting polymer brush for preparing PDMS-KH570-hydrogel-PSBMA samples were 20 and $120 \mathrm{~min}$, respectively. 

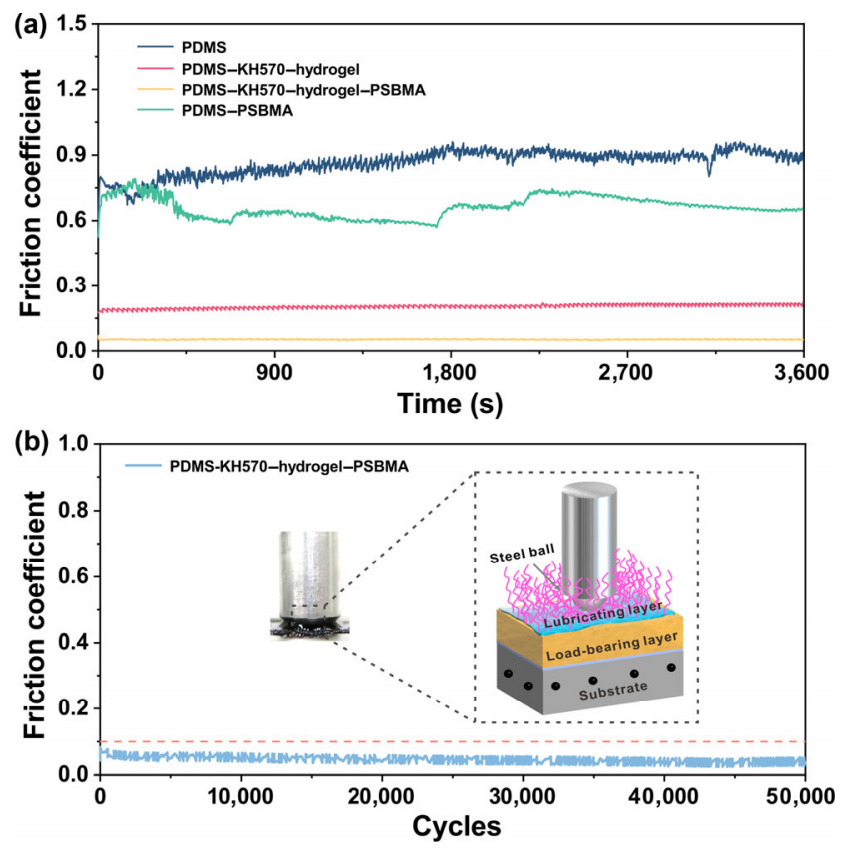

Fig. 9 (a) Evolution of COF with time for PDMS, PDMSKH570-hydrogel, PDMS-KH570-hydrogel-PSBMA, and PDMSPSBMA (Applied load: $5 \mathrm{~N}$ ) and (b) COF-cycles curve on the surfaces of PDMS-KH570-hydrogel-PSBMA for 50,000 friction cycles at applied load of $10 \mathrm{~N}$. The reciprocating frequency of all the above friction tests were $1 \mathrm{~Hz}$. The growth time of hydrogel and grafting polymer brush were 20 and $120 \mathrm{~min}$, respectively.

of $\mathrm{COF}$, and the COF had not exceeded 0.1 all the time, indicating that the polymer brush had not been damaged and failed under the continuous shear of high load. Thereby, it demonstrated that the PDMSKH570-hydrogel-PSBMA exhibited great abrasion resistance.

\subsection{Evaluation of wear-resistance property}

In terms of the wear behavior, worn area and worn scar morphologies were investigated in Fig. 10. By comparing the worn area for PDMS-KH570-hydrogel and PDMS-KH570-hydrogel-PSBMA, it was obvious that the worn depth and area sharply decreased after modification with zwitterionic polymer brush, the worn area was decreased from 41,749 to $5,544 \mu^{2}$ and the depth was decreased from 108 to $49 \mu \mathrm{m}$ (Figs. 10(a) and 10(b)). Additionally, from the worn surface morphologies, it could be seen clearly that the PDMSKH570-hydrogel-PSBMA exhibited a better anti-wear performance than the PDMS-KH570-hydrogel, and there was no significant abrasion trace or wear debris on the surface of PDMS-KH570-hydrogel-PSBMA
(Fig. 10(c)). By contrast, the surface of PDMS-KH570hydrogel was rough and presented evident furrows (Figs. 10(d) and 10(e)). What's more, the long-time anti-wear property was also investigated. As shown in Fig. 10(f), the wear depth and area were 98 and $41,762 \mu^{2}$ for PDMS-KH570-hydrogel-PSBMA after 50,000 cycles, respectively. Relatively, it's a huge wear area. However, the polymer brush could be still observed on the worn surface in Figs. 10(g) and 10(h). It can be inferred that the polymer brush on the surface was worn but not completely worn off after 50,000 cycles. This implied that the PDMS-KH570hydrogel-PSBMA had the anti-wear property.

\section{Conclusions}

In this work, we integrate soft and hydrophilic polyzwitterionic polyelectrolyte brush into the subsurface of the atom transfer radical polymerization (ATRP) initiator-embedded high strength hydrogel layer to generate robust layered soft matter lubrication coating on the surface of PDMS silicone elastomer. The layered lubrication coating is covalently anchored onto the surface of the PDMS by employing surface catalytically initiated radical polymerization (SCIRP) method and exhibits high interface combination force of $120 \mathrm{~N} / \mathrm{m}$. Compared with bare PDMS and polymer brush-grafted PDMS samples, the existing layered lubrication coating endows the PDMS substrate with excellent load-bearing and low friction properties when low viscosity water is used as lubricant. Meanwhile, the topmost brush/hydrogel composite layer is highly effective for aqueous lubrication, while the stiff hydrogel layer with controllable thickness provides high load-bearing capacity. The optimal lubrication performance can be realized by controlling the thickness and modulus of the stiff hydrogel layer, or regulating the embedded ATRP initiator concentration and grafting polymerization time of polyzwitterionic polyelectrolyte brush. Furthermore, the effects of frequencies and applied loads on lubrication property of the layered lubrication coating are investigated systematically. Surprisingly, the long-time friction test result indicates that the coating modified PDMS can maintain a stable low friction level (friction coefficient $(\mathrm{COF})<0.05)$ under the normal load of $10 \mathrm{~N}$ even encountering 50,000 sliding cycles. Finally, the surface 

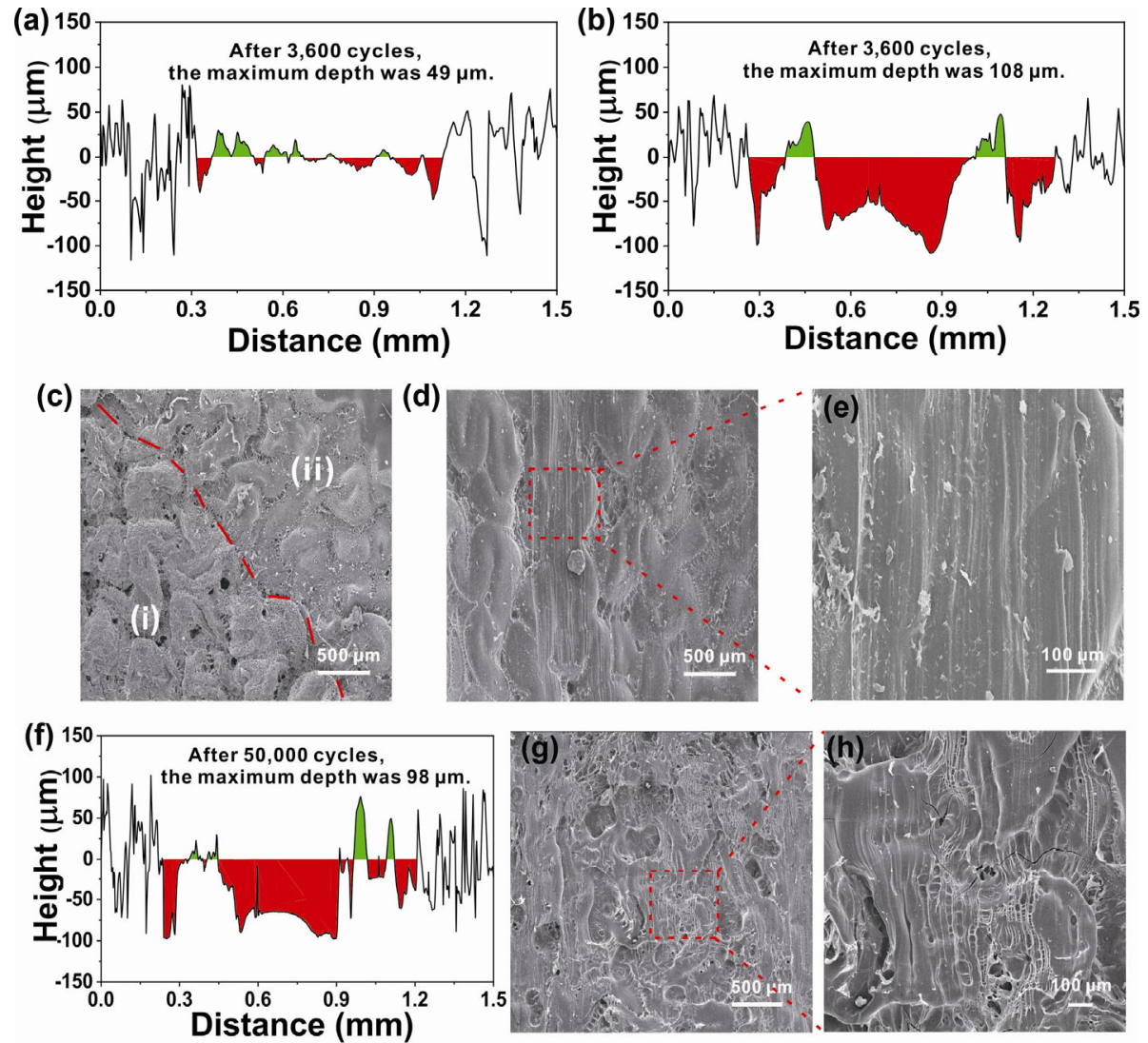

Fig. 10 Cross-sectional profiles of wear scars: (a) PDMS-KH570-hydrogel-PSBMA, (b) PDMS-KH570-hydroge after encountering 3,600 sliding cycles, and (f) PDMS-K570-hydrogel-PSBMA after encountering 50,000 sliding cycles. The SEM micrographs of worn scars: (c) PDMS-KH570-hydrogel-PSBMA ((i) the rubbed area and (ii) the non-tested area)), (d, e) PDMS-KH570-hydrogel after encountering 3,600 sliding cycles, and (g, h) PDMS-KH570-hydrogel-PSBMA after encountering 50,000 sliding cycles.

wear characterizations prove the robustness of the layered lubricating coating. Current work provides important route for preparing lubricious silicone elastomer medical devices with low friction, high load-bearing capacity, and considerable durability.

\section{Acknowledgements}

We gratefully acknowledge supports from the National Key Research and Development Program of China (2016YFC1100401) and National Natural Science Foundation of China (22032006, 52075522). F. Zhou thanks to the project support of the Key Research Program of the Chinese Academy of Sciences (XDPB2404). S. Ma thanks to the support by Youth Innovation Promotion Association (2019411).

Electronic Supplementary Material: Supplementary material is available in the online version of this article at https://doi.org/10.1007/s40544-021-0513-5.

Open Access This article is licensed under a Creative Commons Attribution 4.0 International License, which permits use, sharing, adaptation, distribution and reproduction in any medium or format, as long as you give appropriate credit to the original author(s) and the source, provide a link to the Creative Commons licence, and indicate if changes were made.

The images or other third party material in this article are included in the article's Creative Commons licence, unless indicated otherwise in a credit line to the material. If material is not included in the article's Creative Commons licence and your intended use is not permitted by statutory regulation or exceeds the permitted use, you will need to obtain permission directly from the copyright holder.

To view a copy of this licence, visit http://creativecommons.org/licenses/by/4.0/. 


\section{References}

[1] Yoda R. Elastomers for biomedical applications. J Biomater Sci Polym Ed 9(6): 561-626 (1998)

[2] Jeong S H, Zhang S, Hjort K, Hilborn J, Wu Z. PDMS-based elastomer tuned soft, stretchable, and sticky for epidermal electronics. Adv Mater 28(28): 5830-5836 (2016)

[3] Abbasi F, Mirzadeh H, Katbab A A. Modification of polysiloxane polymers for biomedical applications: a review. Polym Int 50(12): 1279-1287 (2001)

[4] Mata A, Fleischman A J, Roy S. Characterization of polydimethylsiloxane (PDMS) properties for biomedical micro/nanosystems. Biomed Microdevices 7(4): 281-293 (2005)

[5] Goda T, Konno T, Takai M, Moro T, Ishihara K. Biomimetic phosphorylcholine polymer grafting from polydimethylsiloxane surface using photo-induced polymerization. Biomaterials 27(30): 5151-5160 (2006)

[6] Park J H, Lee K B, Kwon I C, Bae Y H. PDMS-based polyurethanes with MPEG grafts: Mechanical properties, bacterial repellency, and release behavior of rifampicin. J Biomater Sci Polym Ed 12(6): 629-645 (2001)

[7] Kai D, Prabhakaran M P, Chan B Q, Liow S S, Ramakrishna $\mathrm{S}, \mathrm{Xu} \mathrm{F}$, Loh X J. Elastic poly(epsilon-caprolactone)polydimethylsiloxane copolymer fibers with shape memory effect for bone tissue engineering. Biomed Mater 11(1): 015007 (2016)

[8] Pinto S, Alves P, Matos C M, Santos A C, Rodrigues L R, Teixeira J A, Gil M H. Poly (dimethyl siloxane) surface modification by low pressure plasma to improve its characteristics towards biomedical applications. Colloids Surf B Biointerfaces 81(1): 20-26 (2010)

[9] Liu L, Sheardown H. Glucose permeable poly (dimethyl siloxane) poly (N-isopropyl acrylamide) interpenetrating networks as ophthalmic biomaterials. Biomaterials 26(3): 233-244 (2005)

[10] Chawla K, Lee S, Lee B P, Dalsin J L, Messersmith P B, Spencer N D. A novel low-friction surface for biomedical applications: modification of poly(dimethylsiloxane) (PDMS) with polyethylene glycol(PEG)-DOPA-lysine. J Biomed Mater Res A 90(3): 742-749 (2009)

[11] Tanaka M, Kurosawa S. Surface Modification of PDMS and plastics with zwitterionic polymers. J Oleo Sci 66(7): 699-704 (2017)

[12] Liu G Q, Cai M R, Feng Y G, Wang X L, Zhou F, Liu W M. Photothermally actuated interfacial hydration for fast friction switch on hydrophilic polymer brush modified PDMS sheet incorporated with $\mathrm{Fe}_{3} \mathrm{O}_{4}$ nanoparticles. Chem Commun 52(18): 3681-3683 (2016)
[13] Lanigan J L, Fatima S, Charpentier T V, Neville A, Dowson $\mathrm{D}$, Bryant M. Lubricious ionic polymer brush functionalised silicone elastomer surfaces. Biotribology 16: 1-9 (2018)

[14] Hemmilä S, Cauich-Rodríguez J V, Kreutzer J, Kallio P. Rapid, simple, and cost-effective treatments to achieve long-term hydrophilic PDMS surfaces. Appl Surf Sci 258(24): 9864-9875 (2012)

[15] Kaneko D, Tada T, Kurokawa T, Gong J P, Osada Y. Mechanically strong hydrogels with ultra-low frictional coefficients. Adv Mater 17(5): 535-538 (2005)

[16] Gong J P, Kurokawa T, Narita T, Kagata G, Osada Y, Nishimura G, Kinjo M. Synthesis of hydrogels with extremely low surface friction. $J$ Am Chem Soc 123(23): 5582-5583 (2001)

[17] Parada G A, Yuk H, Liu X Y, Hsieh A J, Zhao X H. Impermeable robust hydrogels via hybrid lamination. $A d v$ Healthc Mater 6(19): 1700520 (2017)

[18] Yu Y, Yuk H, Parada G A, Wu Y, Liu X Y, Nabzdyk C S, Zhao X H. Multifunctional "hydrogel skins" on diverse polymers with arbitrary shapes. Adv Mater 31(7): 1807101 (2019)

[19] Ma S H, Yan C Y, Cai M R, Yang J, Wang X L, Zhou F, Liu W M. Continuous surface polymerization via Fe(II)mediated redox reaction for thick hydrogel coatings on versatile substrates. Adv Mater 30(50): 1803371 (2018)

[20] Benetti E M, Spencer N D. Using polymers to impart lubricity and biopassivity to surfaces: Are these properties linked? Helv Chim Acta 102(5): 1900071 (2019)

[21] Dehghani E S, Ramakrishna S N, Spencer N D, Benetti E M. Controlled crosslinking is a tool to precisely modulate the nanomechanical and nanotribological properties of polymer brushes. Macromolecules 50(7): 2932-2941 (2017)

[22] Rong M M, Liu H, Scaraggi M, Bai Y Y, Bao L Y, Ma S H, Zhou F. High lubricity meets load capacity: Cartilage mimicking bilayer structure by brushing up stiff hydrogels from subsurface. Adv Funct Mater 30(39): 2004062 (2020)

[23] Husseman M, Malmstrom E E, Mcnamara M, Mate M, Mecerreyes D, Benoit D G, Hedrick J L, Mansky P, Huang E, Russell T P, Hawker C J. Controlled synthesis of polymer brushes by "living" free radical polymerization techniques. Macromolecules 32(5): 1424-1431 (1999)

[24] Du T, Li B, Wang X L, Yu B, Pei X W, Huck W T S, Zhou F. Bio-inspired renewable surface-initiated polymerization from permanently embedded initiators. Angew Chem-Int Edit 55(13): 4260-4264 (2016)

[25] Van Der Heide E, Zeng X Q, Masen M A. Skin tribology: Science friction? Friction 1(2): 130-142 (2013)

[26] Chen M, Briscoe W H, Armes S P, Klein J. Lubrication at physiological pressures by polyzwitterionic brushes. Science 323(5922): 1698-1701 (2009) 
[27] Wang Z N, Li J J, Liu Y H, Luo J B. Synthesis and characterizations of zwitterionic copolymer hydrogels with excellent lubrication behavior. Tribol Int 143: 106026 (2020)

[28] Jiang S Y, Cao Z Q. Ultralow-fouling, functionalizable, and hydrolyzable zwitterionic materials and their derivatives for biological applications. Adv Mater 22(9): 920-932 (2010)

[29] Klein J. Hydration lubrication. Friction 1(1): 1-23 (2013)

[30] Wei Q B, Cai M R, Zhou F, Liu W M. Dramatically tuning friction using responsive polyelectrolyte brushes. Macromolecules 46(23): 9368-9379 (2013)

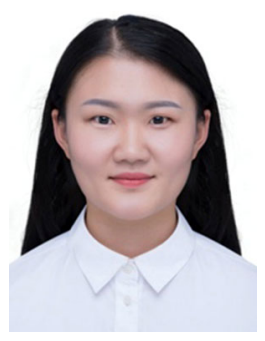

Luyao GAO. She obtained her bachelor degree in 2016 from Ningxia University, China. She is now a Ph.D. candidate in Lanzhou University

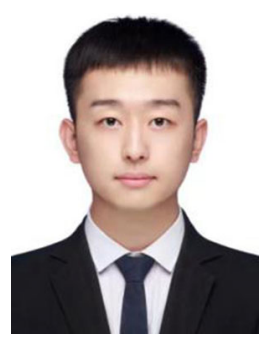

Xiaoduo ZHAO. He obtained his B.S. and Ph.D. degrees in the School of Materials Science and Engineering from Nanjing University of Science and Technology, China, in 2013 and 2020, respectively. Then, he joined

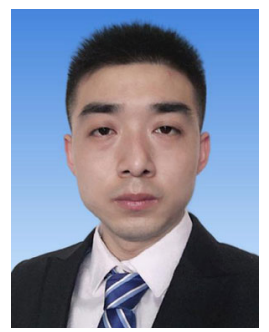

Shuanhong MA. He received his bachelor degree from Tianshui Normal University in 2011 and Ph.D. degree from LICP, CAS in 2016. He became an associate professor of State Key Laboratory
[31] Hower J C, Bernards M T, Chen S F, Tsao H-K, Sheng Y-J, Jiang S Y. Hydration of "nonfouling" functional groups. J Phys Chem B 113(1): 197-201 (2009)

[32] Briscoe W H, Titmuss S, Tiberg F, Thomas R K, Mcgillivray D J, Klein J. Boundary lubrication under water. Nature 444(7116): 191-194 (2006)

[33] Lin P, Ma S H, Wang X L, Zhou F. Molecularly engineered dual-crosslinked hydrogel with ultrahigh mechanical strength, toughness, and good self-recovery. Adv Mater 27(12): 2054-2059 (2015)

and Lanzhou Institute of Chemical Physics (LICP). Her current research interests mainly focus on organic synthesis of biomacromolecules and polymers-based wet/adhesion/lubrication materials.

the State Key Laboratory of Solid Lubrication, LICP, Chinese Academy of Science (CAS), as a research assistant. His research areas cover the surface modification of biomedical polymer materials, tribology, and wear mechanism of hydrogels.

of Solid Lubrication (LSL) in 2018. He published more than 60 papers ( $>1,400$ citations) and applied for 20 patents. His current research interests include surface/interface lubrication modification, synthetic biolubricants, cartilage lubricating materials, and intelligent lubrication devices. 


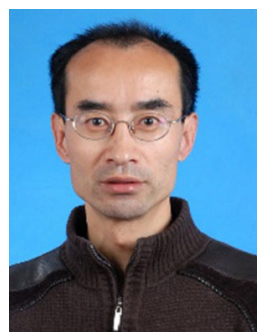

Yong-min LIANG. He received his B.S. degree from Shanxi Normal University in 1989, and M.S. and Ph.D. degrees from Lanzhou University in 1992 and 1998, respectively. He became an associate professor of Lanzhou University (2000-2003). After his postdoctoral

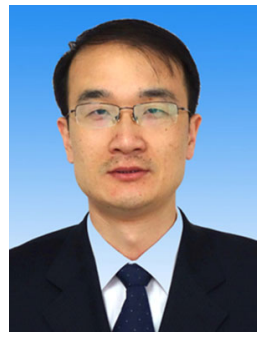

Feng ZHOU. He received his Ph.D. degree from LICP, CAS in 2004. After his postdoctoral research experience at Cambridge University (2005-2008), he became a full professor in 2008 at LICP. He has published more than 400 papers research experience at National Tsing-Hua University (Hsinchu, Taiwan, People's Republic of China) with Professor Show-An Chen (2001-2002), he was promoted to full professor in 2003 at Lanzhou University. His current research interests are the synthesis and application of new functional organic materials and the development of new transition-metal-catalyzed cascade reactions, and new radical chemistry.

(> 19,000 citations) and applied for 140 patents. He is the editorial board members for journals of Friction and Tribology International. His current research interests include surface/interface of soft matters, marine antifouling, drag reduction/noise reduction, biological lubrication, and high performance lubricants. 